\title{
A Case of Foreign-Body Granuloma of the Glabella due to Polyacrylamide Filler and an Intractable Ulcer after Skin Biopsy: An Immunohistochemical Evaluation of Inflammatory Changes
}

\author{
Kio Park ${ }^{a}$ Fuyuko Nishiwaki ${ }^{a, b} \quad K^{b e n j i ~ K a b a s h i m a ~}{ }^{c} \quad$ Yoshiki Miyachi $^{c}$ \\ ${ }^{a}$ Department of Dermatology, Yamato Takada Municipal Hospital, Nara, ${ }^{b}$ Department of \\ Dermatology, Kitano Hospital, Osaka, and 'Department of Dermatology, Kyoto University \\ Graduate School of Medicine, Kyoto, Japan
}

\section{Key Words}

Foreign-body granuloma · Immunohistochemical evaluation · Inflammatory change ·

Polyacrylamide filler

\begin{abstract}
Introduction: Polyacrylamide hydrogel has been considered a safe and biocompatible soft tissue filler, and it has been widely used in cosmetic procedures. However, recent studies have revealed some complications with polyacrylamide filler injections. Case Report: We present the case of foreign-body granulomas of the glabella, which subsequently formed an infectious ulcer 3 years after a polyacrylamide injection. An immunohistochemical evaluation of the foreign-body granulomas was performed in order to study the relationship between foreign-body granulomas and immune response. Conclusion: We believe that our analysis of foreign-body granulomas 1 and 3 years after a filler injection may contribute to revealing the mechanism of chronic and intractable infections after filler injections.
\end{abstract}




\section{Introduction}

Soft tissue augmentation is increasing in popularity as a result of increased interests in facial contouring, breast augmentation, and cheek reshaping [1]. In aesthetic and reconstructive surgery, facial soft tissue augmentation is performed using several biomaterials. Among them, polyacrylamide hydrogel is widely used in cosmetic procedures as a filler material [2]. It is of note, however, that polyacrylamide hydrogel may not be safe or biocompatible [1-4]. Here, we describe a patient who developed foreign-body granulomas on her glabella after an injection of polyacrylamide gel. We also present the results of an immunohistochemical analysis.

\section{Case Report}

A 57-year-old woman was referred to our hospital with a 3-month history of swelling of her glabella. The patient stated that the swelling had become rigid and indurated. Examination revealed a tumor of the glabella (fig. 1a). The tumor was $2 \mathrm{~cm}$ in diameter and was not well demarcated; it was skin colored and caused no redness, pain, or heat. The patient's medical history was unremarkable, with no previous cosmetic interventions. An ultrasound examination of the tumor revealed hypoechoic deposits predominating in the subcutaneous tissue of the glabella and dorsum of the nose (fig. 2). A skin biopsy was performed for diagnostic purposes. Examination of the specimen revealed granulomas consisting of histiocytes and foreign-body giant cells with patchy infiltration of lymphocytes and fibroblasts. Amorphous materials were embedded in the giant cells (fig. 1c). We asked the patient again if she had received a cosmetic filler injection. She finally admitted that she had had a polyacrylamide gel injection in the glabella 1 year before. We diagnosed her with foreign-body granulomas of the glabella due to polyacrylamide filler.

As there remained a slight induration along the surgical scar, the patient was started on oral tranilast (300 mg per day) and hydrocortisone butyrate $0.1 \%$ ointment for 4 months, which resulted in no improvement. The treatment was discontinued, and the tumor was observed without treatment. One year later, the tumor became swollen and red (fig. 1b). We performed an incisional biopsy for diagnosis and again found foreign-body granulomas with diffuse infiltration of lymphocytes and neutrophils (fig. 1d). After the biopsy, the surgical incision did not close despite suturing, and an ulcer formed on the patient's glabella, which became red and infected with purulent discharge. Although she was treated with cefdinir (300 mg per day) and sulfadiazine silver cream, the ulcer persisted for 5 months and still remained unhealed.

\section{Discussion}

Implantation of biomaterials elicits a foreign-body reaction consisting of monocyte adhesion, differentiation to macrophages, and subsequent macrophage fusion to form foreign-body giant cells. Lymphocytes transiently appear at the implant site during the inflammatory response and influence macrophage behavior directly and indirectly with monocytes, macrophages, and foreign-body granuloma cells; however, the lymphocyte response to biomaterials remains unclear [5]. There is a possibility that giant cell formation is stimulated by a Th1 response via interleukin (IL)-2 and interferon- $\gamma$, whereas a Th2 response downregulates foreign-body granulomas by limiting the number of effective 
macrophages and decreasing the number of giant cells as a result of increased IL-4, IL-5, and IL-6 expression [6]. Using CD4, CD8, and CD68 staining, we conducted an immunohistochemical analysis of the foreign-body granulomas in order to evaluate the relationship between $\mathrm{T}$ cells and foreign-body granulomas. In two different specimens that were excised before and 1.5 years after the excisional biopsy, CD4-positive lymphocytes infiltrated more than CD8positive lymphocytes, especially around amorphous structures and hair follicles (fig. 1e-h), which may be consistent with the above explanation. In addition, CD68-positive macrophages surrounded amorphous structures in the dermis (fig. 1i, j).

In our case, the surgical incision formed an infectious ulcer 3 years after the filler injection. We believe that bacteria were introduced during the injection procedure and formed a persistent and intractable infection on the glabella by forming a biofilm [7-10]. Previous reports put forth that infection may occur late after an injection and may be related to immune responses $[8,9]$. Although we did not examine whether the infiltrating T cells were effector $\mathrm{T}$ cells or regulatory $\mathrm{T}$ cells, persisting effector $\mathrm{T}$ cells can become exhausted and function inefficiently in situ [11]. Although it is limited to a single case and issues remain to be addressed in the future, our immunohistochemical analysis of foreign-body granulomas 1 and 3 years after a filler injection may contribute to revealing the mechanism of chronic and intractable infections after filler injections.

\section{References}

1 Manafi A, Emami AH, Pooli AH, Habibi M, Saidian L: Unacceptable results with an accepted soft tissue filler: polyacrylamide hydrogel. Aesth Plast Surg 2010;34:413-422.

-2 de Bree R, Middleweerd MJ, van der Waal I: Severe granulomatous inflammatory response induced by injection of polyacrylamide gel into the facial tissue. Arch Facial Plast Surg 2004;6:204-206.

-3 Cheng NX, Zhang YL, Luo SK, Zhang XM, Hui L, Chen YL, Sheng SY, Wu DH, Wang HB, Li P: Late hematoma, seroma, and galactocele in breasts injected with polyacrylamide gel. Aesth Plast Surg 2011;35:365-372.

-4 Colombo G, Caregnato P, Stifanese R, Ferrando G: Destructive granulomatous reaction to polyacrylamide lip injection: solution for a complex case. Aesth Plast Surg 2011;35:662-665.

5 Chang DT, Colton E, Matsuda T, Anderson JM: Lymphocyte adhesion and interactions with biomaterial adherent macrophages and foreign body giant cells. J Biomed Mater Res 2009;91:1210-1220.

6 Murray CA, DeKoven J, Spaner DE: Foreign body granuloma: a new manifestation of immune restoration syndrome. J Cutan Med Surg 2003;7:38-42.

7 Ono S, Ogawa R, Hyakusoku H: Complications after polyacrylamide hydrogel injection for soft-tissue augmentation. Plast Reconstr Surg 2010;126:1349-1357.

8 Khedher NB, David J, Trop I, Drouin S, Peloquin L, Lalonde L: Imaging findings of breast augmentation with injected hydrophilic polyacrylamide gel: patient reports and literature review. Eur J Radiol 2011;78:104111.

-9 Prantl L, Fichtner-Feigl S, Hofstaedter F, Lenich A, Eisenmann-Klein M, Schreml S: Flow cytometric analysis of peripheral blood lymphocyte subsets in patients with silicone breast implants. Plast Reconstr Surg 2008;121:25-30.

-10 Christensen LH: Host tissue interaction, fate, and risks of degradable and nondegradable gel fillers. Dermatol Surg 2009;35:1612-1619.

11 Virgin HW, Wherry EJ, Ahmed R: Redefining chronic viral infection. Cell 2009;138:30-50. 
Park et al.: A Case of Foreign-Body Granuloma of the Glabella due to Polyacrylamide Filler
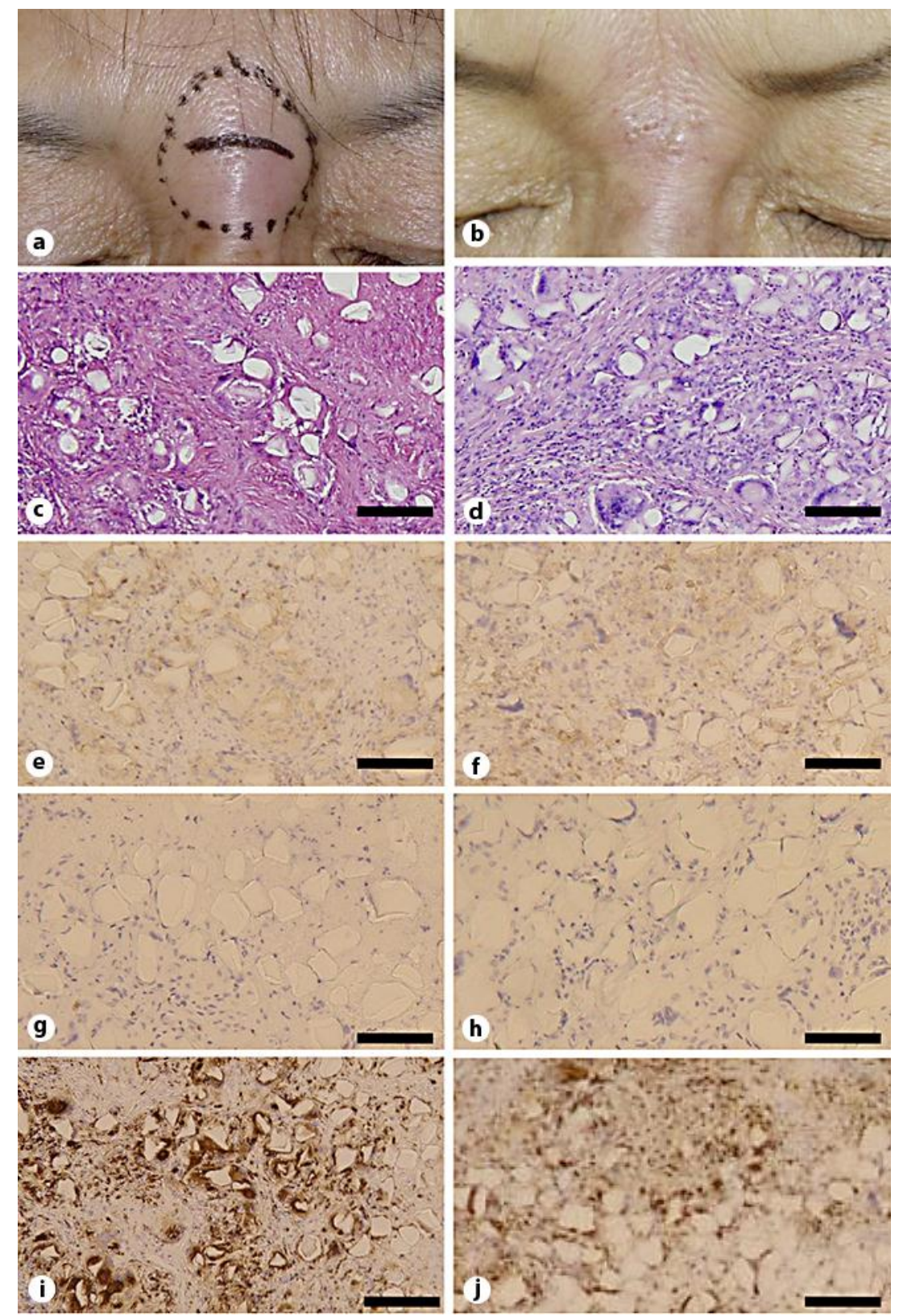

Fig. 1. Clinical and histological findings. a, b Clinical appearance. c-j Histological findings. c, d Hematoxylin and eosin staining. e, f CD4. g, h CD8. i, j CD68. These findings were observed before (a, c, e, g, i) and after (b, $\mathbf{d}, \mathbf{f}, \mathbf{h}, \mathbf{j})$ biopsy. Bars $=10 \mu \mathrm{m}(\mathbf{c}-\mathbf{j})$. 


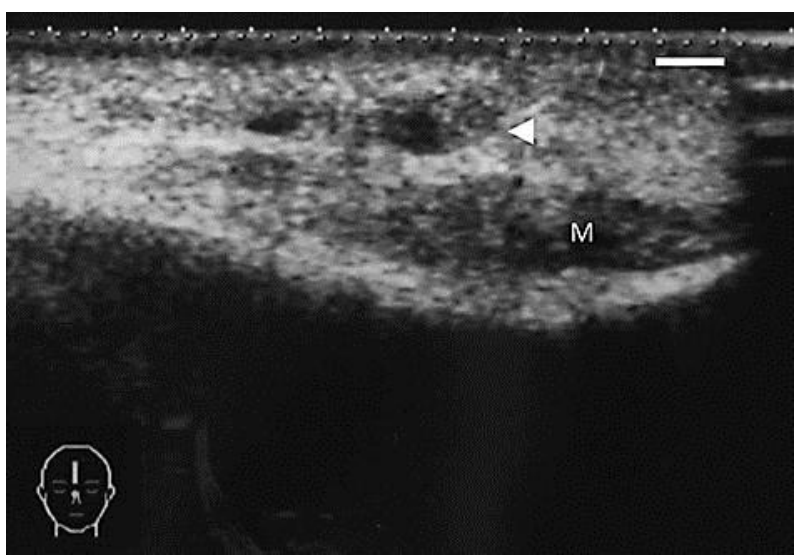

Fig. 2. Ultrasound examination of the glabella demonstrates the low echoic area (arrowhead) above the frontal muscle (M). Bar = $1 \mathrm{~mm}$. 